\title{
Entreprendre une anthropologie des migrations : retour sur un terrain
}

Undertaking an Anthropology of Migration: Reflections on a Fieldwork

Emprender una antropología de las migraciones: reflexiones sobre un trabajo de campo

Catherine Quiminal

\section{(2) OpenEdition}

Journals

Édition électronique

URL : https://journals.openedition.org/remi/4992

DOI : $10.4000 /$ remi.4992

ISSN : $1777-5418$

Éditeur

Université de Poitiers

Édition imprimée

Date de publication : 1 décembre 2009

Pagination : 115-132

ISBN : 978-2-911627-53-8

ISSN : 0765-0752

Référence électronique

Catherine Quiminal, « Entreprendre une anthropologie des migrations : retour sur un terrain », Revue européenne des migrations internationales [En ligne], vol. 25 - n³ | 2009, mis en ligne le 01 décembre

2012, consulté le 15 avril 2022. URL : http://journals.openedition.org/remi/4992 ; DOI : https://doi.org/ 10.4000/remi.4992 


\section{Entreprendre une anthropologie des migrations :

\author{
retour sur un terrain
}

\section{Catherine QUIMINAL*}

L'approche anthropologique des migrations en France est tardive, elle reste encore marginale. Je n'examinerai pas dans ce texte l'ensemble des raisons permettant d'expliquer ce fait ; je me contenterai d'en énoncer une qui n'est pas des moindres. Dans une conjoncture d'ethnicisation, voire de racisation il est périlleux d'isoler un groupe, souvent, lorsqu'il s'agit de travailleurs immigrés, originaire des anciennes colonies, sans contribuer à ce processus d'ethnicisation des rapports sociaux, sans fabriquer de la distance culturelle. Autrement dit étudier un groupe particulier de migrants nécessite de rompre avec une conception englobante, discriminante de la culture, à l'inverse de considérer le caractère plastique, problématique de chaque culture, des cultures mises en présence (Augé, 1994). Le groupe des migrants, lui-même non homogène, ne représente pas « une » culture et ne peut être saisi que dans les relations qu'il entretient avec les siens en France et dans son pays d'origine, avec ceux qui pour lui constituent « les autres » en situation migratoire. C'est donc dans la perspective d'une anthropologie « des mondes contemporains » que je me suis inscrite afin de saisir des dynamiques sociales, des processus d'acculturation et les conflits qu'ils entraînent, mettant au cœur de mes préoccupations les acteurs sociaux, leur interprétation et leur construction du social, sans pour autant négliger les contraintes qui pèsent sur ces dynamiques, les obstacles aux changements. À définir son objet dans son mouvement, ici et là-bas, dans son histoire, dans une situation donnée, situation nationale post-coloniale, toutes les difficultés ne sont pas pour autant résolues. À savoir : à quelles conditions l'anthropologue a-t-il légitimité à travailler sur le proche, dans sa propre société ? Comment construire le groupe stratégique ? Que se passe-il quand le proche est aussi le lointain?

Paradoxalement, liés à la mobilité des migrants, à leurs pratiques se déployant sur plusieurs espaces, ce sont les formes d'investigation caractéristiques de la démarche anthropologique, observation participante et enquête de terrain prolongée qui m'ont amenée à travailler sur un espace recomposé n'opposant pas radicalement ici et là-bas.

\footnotetext{
* Professeure émérite, Université Paris Diderot, URMIS ; catherine.quiminal@univ-paris-diderot.fr
} 
Cet article relate une expérience collective d'enquête anthropologique associant au plus près l'ethnologue et ses sujets d'observation, jouant tout au long de son déploiement sur la réciprocité des regards dans l'objectif de réduire au maximum le procès d'altérisation.

Elle a privilégié une connaissance de l'intérieur (Althabe, 1990) en étroite collaboration avec certains acteurs de la migration soninkée en France, une approche anthropologique, nécessaire à la compréhension de situations qui ne cessent de se multiplier en raison de la mondialisation. Menée dans les années quatre-vingt, sur une longue période, elle préfigure, en quelque sorte, les réflexions sur les recherches multisites, sur le transnational, d'ethnologues américains (Marcus², 1986 ; Glick Schiller et al., 1992 ; Appadurai, 1996, pour ne citer qu'eux) interrogeant la culture comme paradigme central, repositionnant leurs « objets » comme des participants contemporains de systèmes de pouvoir économiques et sociaux pris dans la mondialisation.

\section{LE FOYER POUR TRAVAILLEURS IMMIGRÉS COMME UNIVERS DE RÉFÉRENCE. LA DOUBLE CRISE}

L'anthropologie peut être conçue comme un « déplacement » du relatif à l'universel ou comme un détour, un va-et-vient entre soi et l'autre ; les enjeux de connaissance sont différents dans les deux cas. Dans le deuxième cas, le projet du chercheur part de l'hypothèse que la distinction entre soi et l'étranger, distinction toujours construite, est constitutive du lien social. La définition de soi par un groupe est toujours définition par rapport à l'autre. C'est ce rapport qui intéresse le chercheur, dans sa pluralité, jusqu'au rapport de sa propre société avec celles des autres.

Pour ma part, avoir comme objet de réflexion une situation migratoire c'était tenter de comprendre un certain nombre de relations induites par cette situation. Au départ mon projet n'était nullement d'étudier les migrations soninkées ${ }^{3}$ en France mais de comprendre les processus d'exclusion interne ou d'intégration générés par le type d'habitat provisoire pour hommes célibataires immigrés qu'étaient les foyers. Les résidents étaient en lutte contre les augmentations de loyer peu compatibles avec leur salaire et les règlements intérieurs peu compatibles avec leur condition d'homme adulte. Certains foyers étaient habités par des Portugais, d'autres par des Maghrébins, d'autres par des « Africains ». Le choix de tel ou tel foyer releva plus de la contingence, pouvoir y mener mon étude en raison des liens tissés antérieurement et en d'autres lieux avec certains résidents, que d'impératifs scientifiques ou liés à la qualité d'africaniste que je n'étais pas. L'unité résidentielle m'avait semblé propice à une approche anthropologique. J'ignorais alors qu'elle me conduirait en Afrique.

2 Le texte de Marcus insiste sur l'intérêt des recherches multisituées pour l'étude des migrations. "Multi-sited ethnographies define their objects of study through several different modes or techniques: - Follow the people. Migrations studies are perhaps the most common contemporary research genre of this basic mode of multi-sited ethnography" (1995 : 96-97).

3 C'est le foyer qui m'a fait découvrir l'existence d'immigrés originaires de la Vallée du fleuve Sénégal en France, les Soninké entre autres. 


\section{Une altérité construite}

On assiste à la fin des années soixante et au début des années soixante-dix à un développement des politiques migratoires. Les lois, décrets régissant flux et stocks se multiplient. Pourtant l'action publique pour le logement des immigrés est presque inexistante guidée par l'idée que les immigrés ne doivent pas s'installer définitivement en France. C'est dans cette optique que les pouvoirs publics vont confier à la Sonacotra la construction de foyers pour salariés célibataires algériens dans un premier temps. Puis face au scandale des marchands de sommeil et des bidonvilles et à la satisfaction des promoteurs immobiliers, ce type de logement va être proposé à des immigrés d'autres nationalités, mais toujours selon une logique ethnico-culturelle, et soucieuse du « retour» des immigrés. «D'abord permettre à ceux qui le souhaitent de sauvegarder leurs liens avec leur culture d'origine. Il y aurait, en effet, intérêt à faciliter le maintien de traditions religieuses, de liens culturels, voire l'expression dans la langue d'origine, de ceux qui souhaitent garder à leur séjour en France un caractère temporaire » (Document préparatoire au Conseil des ministres, 1974) (Weil, 1991 : 96). C'est ainsi que les migrants vont être relogés en fonction de leur origine. Les «Africains » avec les «Africains », les Maghrébins avec les Maghrébins. Cette division spatiale participe de la construction d'une altérité radicale, d'un processus de ségrégation clairement affiché. Il s'agit de rendre invisible, la plus distante possible, une certaine catégorie d'étrangers, des hommes célibataires, simple force de travail qui doit, de surcroît, demeurer bon marché. Dans ces logements temporaires, les résidents n'ont pas le statut de locataire et, en principe, ils n'ont pas le droit d'y habiter plus de deux ans, ils sont régis par des règlements intérieurs conçus pour des hommes pas comme les autres. Interdiction d'aménager les chambrées, aucun meuble personnel n'est toléré, droit de visite interdit... Les gérants, souvent d'anciens coloniaux sont chargés d'y faire régner l'ordre (Samuel, 1975 ; Quiminal, 1991).

\section{Reprise d'initiative}

Le foyer sur lequel j'avais jeté mon dévolu dès le milieu des années soixante-dix, était principalement habité par des Soninké, souvent originaires de la région de Kayes au Mali. Un regroupement aussi sélectif ne relevait pas uniquement de la politique publique. Il résultait également des luttes pour le relogement menées par les migrants contre les taudis dans lesquels ils vivaient (usines désaffectées, caves, immeubles insalubres). Ils revendiquaient d'être relogés collectivement en un même lieu afin de pouvoir continuer à tisser les solidarités nécessaires pour affronter les conditions de précarité qui étaient les leurs.

La présence de nombreux Soninké, parfois cinquante ou soixante originaires du même village, dans un même foyer témoignait en outre d'une forte pression à la migration dans la région. En effet les faiblesses de l'économie d'auto-subsistance entraînaient de plus en plus de jeunes à prendre le chemin de la France : besoins croissants en numéraire, délitement du tissu social, désir d'échapper à la tutelle des aînés. Les cadets supportaient de moins en moins la dépendance à des personnes, le rapport salarial leur apparaissait telle une libération. 
Dès lors il n'est pas étonnant que le foyer ait été le lieu de logiques multiples : celle de la puissance publique, ségrégationniste, celles des résidents, d'aménagement et de valorisation de leur présence en France. Ces logiques mettaient en jeu plusieurs constructions de l'autre, plusieurs constructions identitaires. Pour les migrants la situation était d'autant plus complexe qu'étranger dans la société où ils travaillaient et vivaient, ils risquaient de devenir étrangers au regard de leur société de départ. Pourtant le regroupement en un même lieu d'habitation fut un des facteurs qui leur permit une « reprise d'initiative » notamment au regard de leur village d'origine (Balandier, 1955).

\section{Le système terre/argent revisité}

Le système terre/argent (Quiminal, 1994), mis en place depuis déjà longtemps, qui permettait à l'économie familiale de perdurer en articulant agriculture familiale vivrière et envoi d'argent des migrants était en crise. Les demandes d'envoi d'argent adressées par les patriarches à leurs migrants se faisaient de plus en plus importantes, l'utilisation de cet argent incontrôlable et souvent peu légitime au regard des migrants : dépenses somptuaires, dote pour une nouvelle épouse... La suspension de l'immigration économique, interrompant le système de rotation des migrants, un cadet remplaçant un aîné - l'accroissement du chômage et le changement des comportements dû à une insertion prolongée des migrants dans le salariat, furent autant de facteurs qui amenèrent ces derniers à porter un regard critique sur cette économie d'autosubsistance qu'ils s'épuisaient à maintenir debout alors qu'ils l'avaient quittée. Par le biais d'associations, les migrants se proposaient de briser un cercle devenu insupportable : l'augmentation des besoins en numéraire entraînant celle du nombre de migrants cette dernière entraînant à son tour une diminution de la main-d'œuvre requise pour emplir les greniers...

Ces associations visaient une utilisation plus rationnelle de l'épargne des émigrés, un transfert des connaissances acquises et de certaines normes de consommation. Pour ce faire elles entendaient constituer le village et non plus le patriarche de la famille comme interlocuteur de leurs initiatives : coopérative d'achat, école, dispensaire, petit barrage. De manière insidieuse, mesurée, la soumission aux anciens était contestée. Les émigrés entendaient assumer leurs devoirs, leurs responsabilités familiales plutôt que de se soumettre à des obligations. Ils se considéraient comme co-responsables des besoins familiaux, en droit d'intervenir sur les moyens les plus appropriés pour les satisfaire, légitimes à exercer un contrôle sur l'utilisation de leur salaire. Le respect des anciens, sans cesse réaffirmé n'exprimait plus, à leurs yeux, la dépendance mais une prise en charge de l'avenir de leur famille par le truchement d'actions collectives à l'échelle du village. De telles initiatives n'allaient pas sans heurter les hiérarchies villageoises. Elles suscitèrent discussions, oppositions, conflits. Les échecs furent nombreux. Ils interrogeaient d'autant plus les migrants que les projets reposaient sur leur contribution financière. Étrangers en France, soupçonnés de trahison au village la menace pesait sur eux d'être " gens de nulle part », pour reprendre une expression utilisée sous la forme d'une question par G. Balandier dans l'article du monde 4 qu'il consacra à mon ouvrage « Gens d'ici Gens d'ailleurs ».

4 Le monde, 18 février 1991, rubrique Sociétés par G. Balandier, « Gens d'ailleurs, gens de nulle part?». 


\section{MIGRANTS ET CHERCHEUSE : INVENTER LES FORMES DE COLLABORATION}

C'est dans cette situation de crise identitaire que me fut adressée la demande par trois leaders associatifs de mener ensemble une recherche ayant comme objectif la compréhension de la distance qui progressivement s'instaurait entre eux et les leurs, entre eux et la société dans laquelle ils vivaient. Leur désir de connaître relevait également des nécessités de l'action, d'une volonté de maîtriser les situations dans lesquelles ils intervenaient. La proposition d'une recherche commune ne venait pas des immigrés en général mais d'un groupe de migrants bien particulier, à la recherche d'une redéfinition identitaire. Il s'agissait d'immigrés qui simultanément avaient conscience d'être devenus différents de ceux restés au village, de certains membres des associations en France, mais n'entendaient pas pour autant nier une partie de leur histoire. Demande de connaissance, demande de reconnaissance. L'entreprise n'allait pas de soi.

La situation que les migrants me proposaient d'analyser avec eux était, à certaines conditions que nous allons maintenant décliner, particulièrement favorable à une investigation ethnologique. Expression d'une double crise, celle de la société française incapable de penser l'avenir de ses étrangers, celle de la société soninkée ayant de plus en plus de mal à se reproduire, les initiatives des migrants se présentaient comme des tentatives d'alternatives aux ordres « prescrits », « l'immigré simple force de travail » versus société française, « l'émigré, dépendant, pourvoyeur d'argent ou traître » versus société soninkée. En tant que telles, elles étaient un terrain privilégié pour une approche dynamique, dans le prolongement des travaux de G. Balandier, mettant en rapport la " société officielle », ses institutions, ses valeurs proclamées, ses signes et ses symboles, avec les relations « d'expressivité » qui s'établissaient entre les différentes instances constitutives de cette même société. Le repérage des tensions, des conflits, à partir de l'étude des pratiques des acteurs sociaux qui utilisaient les alternatives présentes en toute société devait permettre de restituer aux phénomènes sociaux leur complexité et leur mouvement. Nous étions au cœur de processus de changement. Processus d'une extrême complexité puisqu'ils mettaient en jeu les rapports entre la société de départ et la société d'accueil, ceux entre la société de départ et ses émigrés, entre la société d'accueil et ses étrangers, et, à l'intérieur des associations des rapports reproduisant l'ordre villageois et ceux induits par les initiatives.

Notre travail consistait à partir de « l'événement » que constituait notre présence à trouver les médiations entre ces différents univers de communication en pleine mutation. Une recherche collective, menée, partagée avec des migrants en quête de connaissance m'avait semblé propice à construire ces passages. Une telle démarche supposait de s'inscrire dans un temps long nécessaire à une connaissance de l'intérieur, approfondie ; elle exigeait également de redéfinir au fil des événements les frontières d'un même terrain.

La demande que me faisaient les migrants témoignait d'une relative confiance acquise en raison de ma présence prolongée dans le foyer, bravant la chasse qu'un gérant particulièrement zélé pour faire respecter le règlement menait contre moi, me traitant de prostituée, me menaçant de lâcher ses chiens policiers. Cette confiance - outre le respect que je témoignais aux résidents à travers l'intérêt que je portais à l'organisation de leur vie 
quotidienne, à leur pays, à leur histoire - reposait indéniablement aussi sur la position que j'avais prise lors de la lutte des foyers SONACOTRA. À l'origine du Comité de vigilance contre les expulsions des dirigeants de la grève des foyers ${ }^{5}$, active dans les permanences anti-raciste, la nature de mon engagement citoyen n'était pas étrangère à cette confiance. Il convient de souligner que ce préalable à la relation d'enquête fut un élément constant de tous mes terrains, notamment plus tard celle concernant les femmes africaines qui m'avaient surnommée « la chercheuse africaine » (Quiminal, 1998) n'acceptant pas les relations que dessinaient ce qu'elles nommaient un « colonialisme doux ». Mon positionnement clairement anti-raciste constituait un premier pas à mon intériorité à la situation d'enquête, me donnait accès à des faits ordinairement cachés à l'étranger.

Plusieurs moments ont scandé cette expérience de travail en commun. Tous furent occasions de discussions, négociations serrées et propres à chaque étape de la recherche.

\section{Redéfinition du terrain et discussion de la problématique}

Comme je l'ai signalé plus haut je n'avais pas vocation à mener mes recherches en terrains africains. C'est d'une part la situation et d'autre part l'insistance des migrants partie prenante du projet qui m'ont conduite dans les villages de la région de Kayes. « Tu ne peux comprendre qui l'on est, ce qu'on fait si tu ne connais pas d'où l'on vient » me disaient-ils pour m'inciter à parcourir en sens inverse le chemin qui avait été le leur. L'univers de référence des migrants se déployait sur deux espaces. Le terrain s'imposait dans sa double dimension : ici et là-bas, les deux lieux où se déroulait la vie des migrants, au sein desquels ils construisaient leurs représentations, leurs habitus, leurs stratégies tant locales que de communication d'un espace à l'autre, dans une situation de contraintes multiples. C'est bien à la compréhension de la totalité de cette situation que mes compagnons de réflexion entendaient participer. Les trois migrants demandeurs d'une recherche commune avaient, à travers leur communauté d'expérience de lutte dans les foyers et les associations, atténué grandement leur différence de statut : l'un était issu d'une famille de marabout, l'autre fils d'un chef de village, le troisième descendant d'esclave. Ils affirmaient que leur condition de travailleurs émigrés-immigrés avait aboli les différences antérieures.

Ils m'offraient à comprendre une situation de crise, don, qui, à l'accepter, exigeait que nous réfléchissions aux modalités inédites d'échanges, de réciprocité et de restitution des connaissances. Soulignons que la reconnaissance, la désignation, des intérêts et des destinations réciproques, a été explicitée dès le départ, même si selon les situations, les positions respectives d'acteurs ou de témoins étaient sans cesse à redéfinir.

Décision fut prise de redéfinir le terrain, de mener ensemble les enquêtes auprès d'associations investies dans des projets de transformation villageoise en France et dans les villages auxquels étaient destinés les projets. La connaissance qu'avaient mes compa-

5 Le Comité de vigilance auquel participait notamment S. de Beauvoir, M. Foucault et P. VidalNaquet et bien d'autres intellectuels était perçu par les dirigeants de la grève qui a duré plus de cinq ans (1975-1981) non seulement comme un moyen de prévenir les expulsions mais aussi comme l'occasion de nouer des liens verticaux avec des autochtones. 
gnons de recherche, en tant qu'immigrés, des diverses associations comme celle concernant les villages constituait un capital précieux. Le choix des associations fut l'objet de longs débats portant sur la nature des initiatives à privilégier, école, maternité, coopérative d'achat, l'état de développement du projet, en négociation ou en cours, l'existence connue de conflits au sein de l'association ou dans le village concerné par le projet, enfin la situation de réussite ou d'échec du projet. Ces choix indiquaient les villages où nous devions mener nos investigations. Le savoir commun des trois migrants, partie prenante du projet de recherche, leur « compétence $»^{6}$ sur la société locale, furent là encore un capital de connaissances non négligeable pour prendre nos décisions.

Trois associations donc trois villages furent finalement retenus comme lieux d'investigation approfondie, de longue durée - ce qui ne nous interdisait pas quelques incursions dans d'autres situations significatives pour la recherche. La première a été retenue pour le caractère exemplaire de son initiative. Il s'agissait d'une association qui avait construit une maternité qui fonctionnait relativement bien notamment grâce au retour d'un groupe d'immigrés et d'un programme de sensibilisation mené auprès des femmes. La deuxième avait impulsé un projet agricole privilégiant une agriculture collective sur un terrain dont l'appartenance était revendiquée par deux villages au passé conflictuel. L'initiative s'était soldée par un échec retentissant ayant provoqué mort d'hommes et emprisonnement d'un des initiateurs. La troisième association avait déjà à son actif la création d'une coopérative d'achat, source d'économie pour les villageois. Les discussions en son sein étaient âpres quant à la nature de la prochaine initiative. Fallait-il construire un dispensaire ou une école?

Le choix de ces terrains était étroitement lié à la problématique, à la méthodologie que nous entendions adopter et dont nous discutions parallèlement. En tant qu'anthropologue la place que j'occupais était déterminante, la relation quelque peu inégale. Un accord fut trouvé sur l'appréciation des situations instables, bousculant peu ou prou les hiérarchies, l'ordre villageois. L'étude des textes de G. Balandier, notamment certains passages de «Sens et puissance » constitua un temps fort dans l'élaboration d'une problématique commune. Mes compagnons de réflexion adhérèrent à l'idée que l'ordre dominant n'est jamais un ordre total, définitif, que derrière les permanences formelles s'expriment de manière plus ou moins ouverte des pratiques de résistance, de reconstruction identitaire dans lesquelles ils se reconnaissaient. Armés d'un regard critique sur leur propre société, Ils se convainquirent rapidement de l'intérêt à étudier les stratégies des différents acteurs en présence lors des initiatives afin de comprendre les obstacles auxquels ils se heurtaient, les conflits qu'ils suscitaient, les habitudes qu'ils bousculaient, les autorités qu'ils contestaient, par les dynamiques qu'ils impulsaient, les nouveaux savoir-faire techniques et politiques qu'ils importaient, les nouvelles formes de pouvoir qu'ils tentaient de mettre en place.

La « politique du terrain » (Olivier de Sardan, 1995) retenue fut d'assister aux principaux événements concernant les projets de transformation villageoise impulsés par

6 « Cette compétence ne signifie pas qu'il (l'interlocuteur) soit considéré nécessairement comme un « expert» au sein de la société locale, et encore moins qu'il faille accepter le principe de « l'informateur privilégié », grand érudit sur lequel le chercheur se reposerait pour produire un récit « collectif» (Olivier de Sardan, $1995: 81$ ). 
les associations de migrants en région parisienne, de mener des enquêtes qualitatives, mêlant observation et entretiens avec les acteurs concernés positivement ou négativement en France et dans les villages dans la région de Kayes. L'apprentissage du terrain devait se faire au fil des enquêtes. Les hypothèses exploratoires et les méthodes d'enquêtes établies, nous devions trouver les moyens de financer notre recherche. Reconnaître l'équipe de recherche que nous formions n'allait pas de soi. Les institutions susceptibles de nous financer considéraient que des migrants pouvaient tout au plus être des " informateurs ", non participer à part entière à une recherche. Finalement le projet fut accepté en 1982 dans le cadre d'une Action Thématique Programmée (ATP) du CNRS. Il fut décidé que les crédits, 70000 francs, seraient entièrement consacrés aux frais de missions, aucun de nous ne touchant quelque rémunération que ce soit. Un déséquilibre existait cependant dans la mesure où pour moi il s'agissait de mon travail d'enseignante-chercheuse alors que pour les immigrés c'était un travail en plus de leur travail d'usine, non rémunéré. Le seul avantage financier dont ils bénéficiaient était un billet d'avion pour se rendre sur le terrain qui était toujours proche de leur village. Il aurait été plus sain de pouvoir rémunérer ce travail bien qu'aucun problème n'ait surgi de ce côté, ce qui tient plus à la qualité des intéressés qu'au bien fondé de telles pratiques. Les crédits obtenus, il fallut se mettre au travail.

\section{Pratiques de terrains et confrontation des savoirs}

Les enquêtes auprès des associations en France s'échelonnèrent sur plusieurs années (de 1983 à 1992) entrecoupées par des séjours sur le terrain africain, dans les villages où intervenaient les associations. Le travail sur le terrain se déroula de la manière suivante : En France entretiens, recueils d'informations auprès de responsables des associations retenues, assistance aux assemblées générales, discussions dans les chambres des foyers avec les membres de ces associations. Dans la mesure des possibilités de chacun, nous nous étions donnés comme règle la présence simultanée d'au moins deux d'entre nous lors de chaque moment d'enquête. Cette exigence nous permit de confronter la sélection des données, leurs interprétations.

La participation de responsables d'associations aux recherches fut rapidement connue. Elle légitima ma présence dans des situations habituellement fermées aux regards extérieurs notamment lorsque des conflits importants traversaient les projets. Ma présence en tant que chercheuse eut été considérée comme indiscrète, voire incongrue, lors des débats internes aux communautés villageoises reconstituées en France sans cette « garantie ».

De leur côté, les migrants engagés dans la recherche, confortaient par ma présence la reconnaissance de leurs compétences à diriger des associations. D'une certaine manière chaque protagoniste se servait, bénéficiait de la position de l'autre. Moi de leur intériorité à la situation à étudier, eux de mon extériorité. Ils recherchaient la distance, je recherchais la proximité. II va sans dire qu'au cours de la recherche cette différence de positions lorsque des tensions surgissaient à propos de telle ou telle interprétation servait d'argument d'autorité. L'extranéité était encore utilisée comme si elle supposait une impossibilité de communication, l'implication comme contraire à toute objectivité. 
Outre une présence commune lors des différents événements considérés comme significatifs pour notre recherche, nous nous réunissions au moins une fois par mois pour échanger les informations, les observations recueillies, confronter l'interprétation que nous en faisions, étudier des textes et préciser, reformuler pas à pas nos axes de recherche.

Au Mali la situation d'enquête était plus favorable. Nous pouvions les uns et les autres y consacrer l'intégralité de notre temps qui pour les migrants était celui de leurs vacances! Les villages retenus pour une enquête intensive furent investis collectivement une première fois, puis pour ma part durant plusieurs séjours accompagnée d'au moins un des migrants, enfin à plusieurs reprises seule, dans les villages sélectionnés ou dans des villages concernant des projets nous ayant paru particulièrement significatifs pour aborder notre sujet : migrations et transformations villageoises.

Le partage d'un temps relativement long entièrement dédié à l'enquête menée collectivement représenta un moment privilégié de nos échanges. La diversité des entrées en dialogue, des interactions verbales, le travail collectif, furent une manière d'introduire les débats dans l'enquête au niveau de la production des données et des stratégies interprétatives qui s'y manifestaient. (Olivier de Sardan, 1995). Nous procédions tous les soirs à ces débats sans pour autant nous mettre toujours d'accord sur l'interprétation à donner des observations, des informations, des propos recueillis. En cas de désaccord trop important nous interrogions les différences de référents, de subjectivités sans pour autant nous sentir obligés de trancher sur le degré de véracité des données. La recherche ne pouvait être fructueuse sans un travail, d'autant plus contraignant qu'il était étranger aux migrants, d'appropriation de la littérature savante susceptible d'éclairer nos questionnements.

\section{Formation à l'anthropologie}

À la demande de mes « compagnons de réflexion », la première réunion d'étude porta sur une présentation qu'ils se chargèrent de faire des us et coutumes ayant cours dans leurs villages, des différentes formes de pouvoirs, des différents rapports sociaux et activités. Ils affirmaient par ce choix leur refus d'être coupés de leur histoire, la volonté d'exprimer, de me faire connaître la complexité de leurs appartenances, mais aussi de partager leur connaissance. Cette exigence nous amena à confronter le regard que les migrants portaient sur leur propre société, à celui des chercheurs, consigné dans les livres. Saint-Père, Meillassoux, Pollet et Winter, Chastanet, Weigel, Samuel ${ }^{7}$.

Cette incursion dans la littérature savante, datant dans certains cas de la période coloniale, fut l'occasion pour les migrants de se réapproprier une partie de leur histoire, de la confronter à celle qui leur avait été restituée à travers la mémoire des anciens (Quiminal, 1991). Pour moi ce fut un premier pas vers l'autre, saisi dans une histoire, fragmentaire, dépendante de la vision d'ensemble et des préoccupations de chaque témoin, certes, mais bien présente. Premiers pas livresques sur un terrain déjà défriché qui devaient orienter, grâce aux connaissances disponibles déjà accumulées, l'observation directe. La confrontation des différents savoirs nous permit de repérer ce qui relevait du changement et ce qui relevait du regard des autres, ceux qui nous avaient précédés sur le même terrain, ceux qui

7 Auteurs ayant étudié la société soninkée. Cf. bibliographie. 
étaient des acteurs de la société étudiée. L'existence même de ces différents regards, leur confrontation, constituaient un premier élément de distanciation, une manière d'aborder l'autre à partir d'un univers commun de communication, ne privilégiant ni sa dimension exotique ni sa dimension universelle, une tentative d'établir une relation entre le discours de l'autre et le discours sur l'autre (voir Kilani, 1994). Le fait de multiplier les « niveaux d'extranéité », les migrants étant à la fois mêmes et autres, joua un rôle non négligeable dans notre travail. Les points de vue emic et etic étaient constamment en dialogue.

$\mathrm{Au}$ fur et à mesure que nous avancions dans la connaissance de notre terrain surgissaient de nouveaux problèmes, les textes anthropologiques auxquels nous avions besoin de nous référer se multipliaient. Les principaux travaux d'anthropologues français auxquels je me suis efforcée de donner accès à mes compagnons furent principalement ceux de G. Balandier pour les connaissances qu'ils nous livraient de cette " Afrique ambiguë », pour l'attention qu'ils portaient aux différentes formes de résistance, à la situation coloniale ; ceux de C. Meillassoux pour l'analyse qu'ils proposaient du système international des migrations, l'importance pour ce dernier de pouvoir bénéficier de l'existence menacée des sociétés d'auto-subsistance ; ceux de J.-L. Amselle qui nous invitaient à considérer les migrations comme des processus à replacer dans leur histoire ; ceux de J.-P. Olivier de Sardan à la recherche du mode d'articulation entre les contraintes extérieures et les initiatives paysannes ; enfin ceux de G. Althabe en tant que réflexion sur la méthode anthropologique, sur les risques d'être « prisonnier de la situation de terrain ». L'inédit de notre recherche résidait dans le fait d'avoir abordé l'ensemble des problèmes ici rapidement désignés à partir des situations migratoires et avec certains migrants. La lecture et l'interprétation commune de textes souvent difficiles furent particulièrement édifiantes pour les migrants comme pour moi. Mon rôle de pédagogue fut facilité par le défi et les enjeux que représentait pour mes compagnons l'accès à la théorie.

C'est néanmoins dans le rapport aux connaissances théoriques que les inégalités entre chercheuse professionnelle et migrants étaient les plus importantes ne serait-ce qu'en raison de la familiarité différente avec une telle pratique, du temps dont chacun disposait. Malgré son intérêt, je ne développerai pas ce point ici. Je peux simplement signaler que la préoccupation de partager les savoirs théoriques a été constante et a donné lieu à des échanges d'interprétation aussi féconds qu'étonnants. Notre collaboration impliquait enfin la discussion des résultats de la recherche. Nous déterminions les points sur lesquels chacun d'entre nous devait intervenir sous la forme d'un texte. La production de textes n'a pas été le fait de la seule chercheuse en titre ${ }^{8}$.

Concernant l'essentiel de nos conclusions, dans la mesure où nous les avions élaborées pas à pas ensemble nous parvenions à un accord. Dans les cas où les divergences subsistaient nous décidions de procéder à une restitution dialogique. Pourtant certaines oppositions demeuraient irréductibles, elles donnaient lieux à des conflits dont il nous fallait comprendre les causes.

8 À l'issue de cette expérience un des membres de l'équipe décida de s'inscrire à l'École des Hautes Études en Sciences sociales et y obtint le diplôme de l'EHESS. 


\section{À PROPOS DE QUELQUES DIFFICULTÉS INHÉRENTES AU PROJET}

Afin de ne pas donner une version irénique de cette expérience qui ne fut pas exempte de tensions, il convient d'exposer certains biais, certaines mésinterprétations, certains fourvoiements produits de notre collaboration.

\section{« L'enclicage " ${ }^{9}$}

Ma présence dans les villages n'avait rien d'évident. L'entreprise de connaissance d'une situation n'est pas toujours une cause que tous les acteurs puissent ou veuillent entendre immédiatement. Un des problèmes qui mit du temps à nous apparaître comme central fut celui de la place des émigrés dans leur village. Longtemps critiqués par les anciens, ceux qui avaient quitté le village se sentaient en dette vis-à-vis du chef de famille (Quiminal, 1991). Culpabilité soigneusement entretenue par ces derniers de manière à préserver des liens de dépendance. Les projets d'intervention dans les villages n'étaient pas étrangers, nous l'avons relevé plus haut, à la volonté de faire cesser ce rapport de domination ce qui n'échappait à personne. Ma venue avec des représentants des émigrés n'était pas neutre, elle était ressentie, que je le veuille ou non, comme une prise de position tant par les émigrés que par les femmes ou les anciens. J'étais perçue comme étant du côté des émigrés, chaque groupe social, voire même chaque individu, utilisant à sa manière cet argument. Ma présence était ressentie comme un événement exploitable, comme un enjeu possible. Elle constituait d'autant plus un événement qu'il s'agissait de la venue d'une étrangère dans un univers villageois dans lequel à la différence de ce qui se passe dans un univers urbain, les événements de rencontre ne sont pas construits sur un ordre objectif, mais bien au regard de la communauté (Quiminal, 1998 ${ }^{10}$. Comme le note G. Althabe dans son ouvrage Oppression et Libération dans l'Imaginaire, après avoir défini ce qu'il entend par événement : « II n'est pas imaginable de pouvoir librement passer sur les chemins, sans préciser qui on est, pour quelle cause on est là ».

En ce qui concerne mes compagnons de réflexion, revenir au village avec une femme blanche professeure des Universités renforçait leur autorité, mais aussi accentuait les différences entre eux et les villageois. Deux exemples sont particulièrement significatifs des effets, contrôlés dans le premier cas négligés dans le second, de ma présence à leur côté.

Dans le premier cas ma venue avait été négociée, acceptée par le conseil du

9 Ce terme construit à partir du mot clique est utilisé par Olivier de Sardan pour désigner un biais « redoutable autant qu'inévitable. Le chercheur peut toujours être assimilé, souvent malgré lui, mais parfois avec sa complicité, à une clique ou une faction locale, ce qui offre un double inconvénient. D'un côté il risque de se faire trop l'écho de sa " clique » adoptive et d'en reprendre les points de vue. De l'autre il risque de se voir fermer les portes des autres cliques locales » (1995: 102).

10 Dans ce texte nous analysons notre premier contact avec les villageois qui en guise de salutations d'accueil nous ont rappelé les nombreux méfaits du colonialisme dans le village. 
village et la famille chez qui je logeais. Les émigrés avaient présenté leur retour temporaire comme un séjour dédié à l'enquête, ce qui ne manquait pas de leur conférer un certain prestige. Dans la mesure où il y avait consensus autour de ma présence comprise comme un soutien aux initiatives des émigrés, nous eûmes beaucoup de mal à rencontrer des points de vue hostiles aux projets. Nul ne se sentait autorisé à quelque critique que ce soit. Mon rapport aux migrants générait une sorte de censure. Nous ne récoltions des conflits, au demeurant fort violents, qu'une version édulcorée. De telles situations n'étaient cependant pas insurmontables grâce à une mise en perspective triangulaire. Un détour par les foyers de résidents pour retrouver des membres des familles hostiles aux initiatives des immigrés s'avéra nécessaire. L'étude comparée des différentes versions d'une même situation permettait de contourner ce genre de difficultés, la mise en relation de différentes interprétations dans des lieux de parole différents fut toujours fructueuse.

Dans le second cas notre venue à des fins d'enquêtes, plus particulièrement la mienne n'avait pas été préparée. Arrivée au village la nuit tombée, village dont un des émigrés de notre équipe était ressortissant, ce dernier me proposa provisoirement de dormir avec sa seconde épouse qui n'était pas de « tour » ce soir là. De nombreux conflits, non dénués d'intérêt mais importés, découlèrent de cette situation créée par l'irruption d'une étrangère dans l'univers familial. Faute de précisions préalables sur les raisons de ma présence, toutes les interprétations devenaient possibles, notamment du côté des femmes. Arrivée seule, accueillie, logée par un de mes compagnons dont c'était le village, je ne pouvais être qu'une rivale, une future troisième épouse... Le malentendu nécessita de nombreuses heures de discussions avec les femmes avant d'être levé (Geertz, 1983). Mais en contrepartie, alors que systématiquement ne m'étaient présentés comme acteurs des projets que des hommes le rapport qu'avait fait naître le conflit fictif entre les femmes et moi nous permit de recueillir les points de vue des femmes sur les projets des hommes ; leurs projets collectifs comme leurs projets migratoires personnels. Nous rentrions dans un jeu d'échanges où les femmes m'utilisaient comme intermédiaire pour faire connaître avec plus de force et moins de danger leurs griefs à l'endroit des hommes, leur point de vue sur les projets des hommes, en l'occurrence la construction d'une école conçue comme devant être mixte. Mes compagnons eurent quelques difficultés à accepter ma nouvelle position. Pourtant s'ils voulaient comprendre certains aspects des initiatives des émigrés il leur fallait aussi intégrer le point de vue des femmes ${ }^{11}$. Malgré mes demandes réitérées mes compagnons montraient une forte propension à privilégier les enquêtes auprès des hommes, à négliger le point de vue des femmes d'un moindre intérêt, selon eux, dans les luttes de pouvoir.

Quels que soient les partenaires, des rapports se nouent, fragiles, fluctuants selon les situations, à renégocier à chaque étape de la recherche.

\section{Clivages au sein de l'équipe de recherche}

Tant à propos des observations faites et notées sur mon carnet de terrain, des enquêtes menées ensemble, qu'à propos de l'interprétation des données, il arrivait que

11 Cf. Gens d'Ici Gens d'ailleurs (1991) et notamment le rapport des femmes à l'introduction d'écoles mixtes. 
surgissent des conflits entre mes compagnons et moi-même, conflits non dénués de violence.

Les critiques qui m'étaient opposées commençaient souvent par une accusation de mensonge : falsification de la réalité, fausse interprétation. Dégager les raisons qui présidaient à nos oppositions, lever la suspicion exigeait souvent un certain temps.

Les principales me semblent avoir été de quatre ordres :

- Des " insuffisances" dans le recueil des informations. Ainsi d'un entretien que j'avais eu avec un membre « contestataire » d'une association, et qui s'étant présenté comme appartenant à une famille de marabout avait omis de me signaler qu'il s'agissait aussi d'une famille de grands commerçants. Je cherchai du côté du religieux explication à son opposition, fourvoyée par une grève de mosquée organisée par les villageois en rétorsion à son opposition à la création dune coopérative d'achat. Or c'est au regard des activités marchandes de sa famille qu'il s'opposait à ce projet qui était destiné à réguler les prix. Mes compagnons rectifièrent mon interprétation. La discussion collective des informations permettait de rectifier l'interprétation de certaines données.

- Un désaccord sur l'image renvoyée de la communauté ici ou là-bas. Mes compagnons étaient très attentifs aux effets que pouvait produire dans la situation de racisme en France la mise en avant de telle ou telle pratique, de tel ou tel lien social. Ainsi je rendis compte d'une scène à laquelle j'avais personnellement assisté dans un foyer. À la suite d'une algarade verbale à propos de l'objectif d'un projet avec lequel il n'était pas d'accord, un jeune d'origine captive avait frappé un noble plus âgé que lui. Toute la chambrée était alors intervenue contre le jeune et l'avait taxé d'une forte amende. L'explication de cette « punition », qui m'avait été donnée était qu'un jeune, qui plus est, issu d'une famille d'esclave, avait osé porter la main sur un noble plus âgé que lui. Cet exemple illustrait la persistance dans les foyers de rapports hiérarchiques propres à l'ordre soninké dans les villages. Dans un premier temps le fait même que cette scène se fut déroulée en France dans un foyer fut contesté par mes compagnons : " II n'existe plus de tels rapports entre nous. Nous sommes tous des ouvriers ». Bien évidemment je n'entendais pas céder à propos d'un événement dont j'avais été témoin. La situation ne se débloqua que lorsque mes compagnons acceptèrent de reconnaître que ce qui les bouleversait était la représentation donnée d'eux mêmes focalisée autour d'une féodalité inacceptable (voir Bensa, 1992). À charge pour moi de trouver les moyens de présenter faits et interprétations dégagés de tout jugement de valeur. Exercice difficile qui souvent révèle que les référents implicites du chercheur sont encore et seulement ceux de sa propre société.

- La diffusion de connaissances portant sur des conflits en cours. Dans la mesure où les projets des émigrés remettaient en cause l'ordre villageois il n'était pas étonnant qu'ils suscitent résistance, opposition, hostilité passive ou active. La production d'un texte écrit, figeant en quelque sorte les positions des différents protagonistes, posait problème à ceux pour qui la demande de connaissance était une demande de connaissance pour l'action : 
« Nous sommes en train de résoudre le conflit qui nous oppose au marabout. S'il lit ce texte, ce qui est fort probable, il sera furieux et tout recommencera. " « Tout le monde sait comment il s'est comporté mais quand tu l'écris après le type il est mal. » Le passage à l'écriture était ressenti comme une inscription définitive dans une situation immuable, comme l'arrêt de leur propre histoire, comme une trace que l'on ne pouvait plus effacer. (Les livres et différents textes concernant nos recherches circulent dans les villages en Afrique et dans les foyers en France). Des détours s'avérèrent une fois encore nécessaires. Un accord fut réalisé entre nous sur le fait qu'il n'était pas question de taire le conflit, non seulement au regard des impératifs de la connaissance mais encore au regard des possibles actions futures. Une des raisons des échecs des projets était précisément l'opposition non décelée ou volontairement ignorée de certaines forces sociales. Il fallait néanmoins trouver une manière ouverte, dynamique, d'exposer le conflit en cours sans en figer les différents protagonistes dans des positions jugées irréversibles. Les situations sont parfois tellement connues des villageois qu'aucun artifice, changement des noms, etc. ne réussit à brouiller les pistes.

- II $y$ a enfin ce que l'on me cachait délibérément mais que je décelais par mes propres moyens d'investigation. C'est sans doute ce qui donna lieu aux débats les plus fructueux. À l'étonnement de mes compagnons, succédait une curiosité pour les méthodes de l'anthropologie. Comment avais-je pu comprendre ce que j'avais compris alors qu'ils avaient tenté de censurer l'événement ? Pourquoi ? Inversement je ne cessais de m'étonner de l'aisance avec laquelle mes compagnons s'appropriaient ce travail commun, le prolongeaient en vue d'affronter des situations inédites.

\section{CONCLUSION}

Aujourd'hui ce jeu de reconnaissance mutuelle, progressive et sans cesse à réajuster en fonction des situations se poursuit. De nouvelles informations sont mises en commun. Elles influent sur l'interprétation des données et par voie de conséquence exigent que le travail soit repris. Mais n'est-ce pas après tout une des manières de connaître l'autre, que de travailler ensemble?

La recherche fut l'occasion outre d'une avancée dans la compréhension des situations qui nous intéressaient, d'échanges d'une grande richesse. Ces échanges furent possibles dans la mesure où, selon les événements, les clés des médiations appartenaient respectivement à tel ou tel partenaire. La place que chacun de nous occupait dans la communauté que nous formions variait selon les interlocuteurs. Les rapports hiérarchiques étaient ainsi constamment bouleversés, encore fallait-il le voir et l'accepter. La voie privilégiée d'accès au terrain relevait d'une certaine implication dans le jeu des rapports sociaux, d'une manière particulière de se déplacer en fonction des situations. Elle comportait certains risques, elle tentait néanmoins d'élaborer une connaissance à partir des « gens » et non de la société française comme auto-référence ; ce qui n'est pas sans intérêt lorsqu'on se propose d'étudier les migrants et d'éviter l'alternative assimilation ou rejet, de pratiquer une certaine réciprocité. 
En définitive cette collaboration n'a-t-elle pas été un moyen d'aborder conjointement la question de la place de la connaissance dans les processus de transformations sociales et politiques et celle tout aussi problématique de l'engagement du chercheur? Elle a également été une des manières de faire de l'anthropologie pour autant que la question « qui est l'autre? » constitue le fond du débat anthropologique.

\section{Références bibliographiques}

ALTHABE Gérard (1969) Oppression et libération dans l'imaginaire, Paris, Maspero, 354 p.

ALTHABE Gérard (1990) Ethnologie du contemporain et enquêtes de terrain, Terrain, 14, pp. 126-131.

AMSELLE Jean-Lou (1976) Les migrations africaines, Paris, Maspero, 126 p.

APPADURAI Arjun (1996) Modernity at Large. Cultural dimensions of globalization, Minneapolis, University of Minnesota Press, $224 \mathrm{p}$.

AUGÉ Marc (1994) Pour une anthropologie des mondes contemporains, Paris, Aubier, 195 p.

AUGE Marc (1994) Le sens des autres. Actualité de l'anthropologie, Paris, Fayard, 199 p.

BALANDIER Georges (1955) Sociologie actuelle de l'Afrique noire, Paris, PUF, 529 p.

BALANDIER Georges (1971) Sens et puissance, Paris, PUF, 334 p.

BENSAAlban (1992) Éthique professionnelle et expérience de terrain, Journal des Anthropologues, 50-51, pp. 21-24.

CHASTANET Monique (1983) Les crises de subsistances dans les villages soninké du cercle de Bakel, de 1858 à 1945, Cahiers d'Études Africaines, 89-90, pp. 5-36.

GEERTZ Clifford (1983) Jeu d'enfer. Notes sur le combat de coqs balinais, in Bali : interprétation d'une culture, Paris, Gallimard.

GLICK SCHILLER Nina, BASCH Linda and BLANC-SZANTON Christina (1992) Towards a Transnational Perspective on Migration: Race, Class, Ethnicity, and Nationalism Reconsidered, New York, New York Academy of Sciences.

KILANI Monder (1994) L'invention de l'Autre, Lausanne, Payot, 318 p.

MARCUS George-E. (1986) Contemporary Problems of Ethnography in the Modern World System, in J. Clifford and G.-E. Marcus Eds., Writing Culture, Berkeley, Los Angeles (USA), London (UK), University of California Press, pp. 165-193.

MARCUS George.-E. (1995) Ethnography in/of the World System: The Emergence of Multi-Sited Ethnography, Annual Review of Anthropology, vol. 24, pp. 95-117.

MEILLASSOUX Claude (1975) Femmes Greniers et Capitaux, Paris, Maspero, 251 p.

OLIVIER de SARDAN Jean-Pierre (1995) La politique du terrain, Enquête, 1, pp. 74-112.

OLIVIER de SARDAN Jean-Pierre (1991) Savoirs populaires et agents de développement, in Olivier de Sardan et Paquot Éds., D'un savoir à l'autre : les agents de développement comme médiateurs, Paris, GRET-Ministère de la Coopération, pp. 17-42.

POLLET E. et WINTER G. (1971) La société soninkée, Bruxelles, Université de Bruxelles, p. 566.

QUIMINAL Catherine (1994) Sexes, territoires et exclusion, Journal des Anthropologues, 59, pp. 63-72.

QUIMINAL Catherine (1991) Gens d'ici Gens d'ailleurs, Paris, Bourgois, 223 p.

QUIMINAL Catherine (1998) Familles, projets et capitaux, in B. Schlemmer Éd., Terrains et engagements de Claude Meillassoux, Paris, Karthala, pp. 285-292.

QUIMINAL Catherine (1998) Comment peut-on être africaine en France ?, Journal des Anthropologues, 72-73, pp. 49-61. 
QUIMINAL Catherine (1994) Migrations et coopération internationale : le rôle des immigrés dans les projets de développement et les formes de coopération dans la région du Fleuve Sénégal, Paris, OCDE, pp. 329-335.

SAINT-PÈRE J.-H. (1925) Les Sarakollé du Guidimakha, Paris, Larose.

SAMUEL Michel (1978) Le prolétariat africain noir en France, Paris, Maspero, 262 p.

WEIGEL Jean-Yves (1982) Migrations et production domestique des soninké du Sénégal, Paris, ORSTOM, $134 \mathrm{p}$. 


\title{
Entreprendre une anthropologie des migrations : retour sur un terrain
}

\author{
Catherine QUIMINAL
}

\begin{abstract}
Alors que l'Anthropologie se donne pour objectif la connaissance de l'Autre, des cultures différentes, il est étonnant de constater que la présence de l'autre chez soi n'ait pas suscité plus d'intérêt chez les anthropologues français. Sans doute est-il périlleux, dans une conjoncture d'ethnicisation, voire de racisation d'isoler un groupe souvent originaire des anciennes colonies sans contribuer à ce processus, sans fabriquer de la distance culturelle. Cet article tente de restituer une expérience menée au début des années quatre-vingt associant au plus près l'ethnologue et ses sujets d'observation, jouant tout au long de son déploiement sur la réciprocité des regards dans l'objectif de réduire au maximum le processus d'altérisation. Le premier temps de cet article présente le contexte de double crise dans lequel se situent les migrants : construction d'une altérité radicale à travers la politique concernant le logement des immigrés notamment, crise identitaire des immigrés. Il rend compte ensuite des différentes étapes et des difficultés auxquelles la collaboration entre migrants et chercheurs à donné lieu : définition du terrain, formes des échanges, dits et non dits.
\end{abstract}

\section{Undertaking an Anthropology of Migration: Reflections on a Fieldwork}

\author{
Catherine QUIMINAL
}

While anthropology's objective is the knowledge of the Other and of different cultures, it is surprising that the presence of the Other at home has not attracted more interest among French anthropologists. Without any doubts it is a hard work, in a situation of ethnicisation and racialisation, to isolate a group, often originated from former colonies, without being exposed to the risk to contributing to the social construction of the racial/or ethnic Other. This article attempts to restore an experiment lead in the early eighties associating the anthropologist and his "subjects" of observation, an experiment aiming, throughout its deployment, at minimizing the process of "otherness". We first present the context of dual crisis in which migrants are caught up: on one hand, the construction, in particular through the housing policy of the public authorities, of the radical otherness of the migrants; on the other hand, the identity crisis in which they live. We then report the various steps and challenges that the collaboration between researchers and working migrants has experimented: definition of fieldwork, nature of the interactions, misunderstandings and conflicts. 


\title{
Emprender una antropología de las migraciones: reflexiones sobre un trabajo de campo
}

\author{
Catherine QUIMINAL
}

Dándose la antropología como objetivo el conocimiento del Otro, de culturas diferentes, es extraño observar que la presencia del Otro en nuestra casa no haya suscitado más interés de parte de los antropólogos franceses. Sin duda en una coyuntura de etnicización, más aun de racialización es difícil apartar a un grupo, frecuentemente originario de una antigua colonia, sin correr el riesgo de contribuir a la construcción de un Otro étnico y racial. Este artículo trata de restituir una experiencia llevada a principios de los años ochenta, asociando lo más fuertemente posible el etnólogo y sus «sujetos de observación», manteniendo a lo largo de la experiencia la reciprocidad de las miradas con el objetivo de reducir lo más posible el proceso de alterización. En una primera parte describimos el contexto de la doble crisis que los migrantes deben afrontar: por una parte construcción de una alteridad radical a través de la política pública específica del alojamiento de los migrantes, por otra parte profunda crisis de identidad de estos últimos. Abordamos después las diferentes etapas que permitió la colaboración entre investigadores e inmigrantes: definición del trabajo de campo, naturaleza de los intercambios, limites del dicho y del no dicho. 